\title{
A Solution Study on the Local and Global Structure Changes of Cytochrome c: An Unfolding Process Induced by Urea ${ }^{\dagger}$
}

\author{
I-Jui Hsu, ${ }^{\ddagger}$ Ying-Jen Shiu, ${ }^{\S}$ U-Ser Jeng, ${ }^{*}, \mathfrak{£}$ Tung-Ho Chen, ${ }^{\mathfrak{E}}$ Yu-Shan Huang, ${ }^{\mathfrak{1}}$ Ying-Huang Lai, ${ }^{\mathfrak{f}}$ \\ Ling-Na Tsai," Ling-Yun Jang, ${ }^{£}$ Jyh-Fu Lee, ${ }^{\mathfrak{e}}$ Li-Jiaun Lin, ${ }^{\#}$ Sheng-Hsien Lin, ${ }^{\S}$ and Yu Wang \\ Department of Chemistry, National Taiwan University, Taipei 106, Taiwan, Institute of Atomic and Molecular \\ Sciences, Academia Sinica, P. O. Box 23-166, Taipei 106, Taiwan, National Synchrotron Radiation Research \\ Center, Hsinchu, Taiwan 300, and Industrial Technology Research Institute, Hsinchu, Taiwan 310
}

Received: April 19, 2007

\begin{abstract}
The local and global structural changes of cytochrome $\mathrm{c}$ induced by urea in aqueous solution have been studied using X-ray absorption spectroscopy (XAS) and small-angle X-ray scattering (SAXS). According to the XAS result, both the native (folded) protein and the unfolded protein exhibit the same preedge features taken at $\mathrm{Fe} \mathrm{K}$-edge, indicating that the $\mathrm{Fe}(\mathrm{III})$ in the heme group of the protein maintains a six-coordinated local structure in both the folded and unfolded states. Furthermore, the discernible differences in the X-ray absorption near-edge structure (XANES) of these two states are attributed to a possible spin transition of the $\mathrm{Fe}$ (III) from a low-spin state to a high-spin state during the unfolding process. The perseverance of sixcoordination and the spin transition of the iron are reconciled by a proposed ligand exchange, with urea and water molecules replacing the methionine- 80 and histidine- 18 axial ligands, respectively. The SAXS result reveals a significant morphology change of cytochrome c from a globular shape of a radius of gyration $R_{\mathrm{g}}=$ $12.8 \AA$ of the native protein to an elongated ellipsoid shape of $R_{\mathrm{g}}=29.7 \AA$ for the unfolded protein in the presence of concentrated urea. The extended X-ray absorption fine structure (EXAFS) data unveil the coordination geometries of $\mathrm{Fe}(\mathrm{III})$ in both the folded and unfolded state of cytochrome c. An initial spin transition of $\mathrm{Fe}$ (III) followed by an axial ligand exchange, accompanied by the change in the global envelope, is proposed for what happened in the protein unfolding process of cytochrome $\mathrm{c}$.
\end{abstract}

\section{Introduction}

Since the folding concept of describing the polypeptide chains of a protein in its native structure was introduced, protein folding/unfolding has long been a subject of interest. ${ }^{1}$ Such interest is often revived by advances in technologies on structural probes such as absorption, fluorescence, and circular dichroism (CD), which are often used to monitor the local structure changes of proteins during an unfolding process through denaturants or by elevating the temperature in aqueous solution. ${ }^{2,3}$ Structural information deduced from these optical spectroscopes is, however, somewhat less direct in comparison with that obtained from X-ray diffraction, scattering, and/or absorption, which give a direct probe of the electron density distribution or the molecular structure of a protein.

In a recent decade or so, the great advances in the synchrotron radiation source together with the measurement techniques and data analysis algorithms have made small-angle X-ray scattering (SAXS) a practical and increasingly popular probe for nanostructures. Although with a rather low spatial resolution for electron density in comparison with that of X-ray diffraction, SAXS is, however, very sensitive to the mean electron density

\footnotetext{
† Part of the "Sheng Hsien Lin Festschrift".

* To whom correspondence should be addressed. E-mail: usjeng@ nsrrc.org.tw. Address: National Synchrotron Radiation Research Center, 101 Hsin-Ann Road, Hsinchu Science Park, Hsinchu 30076, Taiwan. Tel: +886-3-578-0281, ext: 7108. Fax: +886-3-5783813.

$\doteqdot$ National Taiwan University.

\& Academia Sinica.

$£$ National Synchrotron Radiation Research Center.

\# Industrial Technology Research Institute.
}

fluctuations on the subnanometer to sub-submicron scale either in the solid or in solution system and can provide adequate information for the study of morphology and interactions of biological macromolecules in solution, including equilibrium or dynamic aspects of protein folding/unfolding. ${ }^{3-6}$ Boffi et al. ${ }^{7}$ and Soldatov et al. $^{8}$ demonstrated that X-ray absorption spectroscopy (XAS) could be a direct probe for revealing the local coordination changes near the iron core of the heme group in cytochrome $\mathrm{c}$ or myoglobin when subjected to the changes in $\mathrm{pH}$ value of the solution. A combined study of SAXS and XAS for correlated global and local structural changes is expected to provide insights into the understanding of protein folding/unfolding and/or binding behaviors. ${ }^{3}$

In this work, XAS and SAXS are applied to study the structural changes of a model protein cytochrome $\mathrm{c}$ induced by a denaturant, urea. The oxidized cytochrome $\mathrm{c}$ from a horse heart is a member of the ubiquitous class of heme proteins; the protein contains 104 residues of amino acids, and a heme group consists of a six-coordinated $\mathrm{Fe}(\mathrm{III})$. The heme group is surrounded by three major helices and two cysteine side chains (Cys 14 and Cys 17), which provide the thioether bridges to the heme group. In a native (folded) state, the $\mathrm{Fe}$ of the heme is bonded to four nitrogen atoms of the porphyrin (Por) plane in a pseudo $C_{4}$ symmetry, with two axial ligands from histidine18 (His-18) and methionine-80 (Met-80), as illustrated in Figure 1. ${ }^{9}$ The changes in the coordination geometry of iron and the changes in the overall morphology are investigated using XAS and SAXS when the unfolding of cytochrome $\mathrm{c}$ takes place by introducing urea into the aqueous solution. 


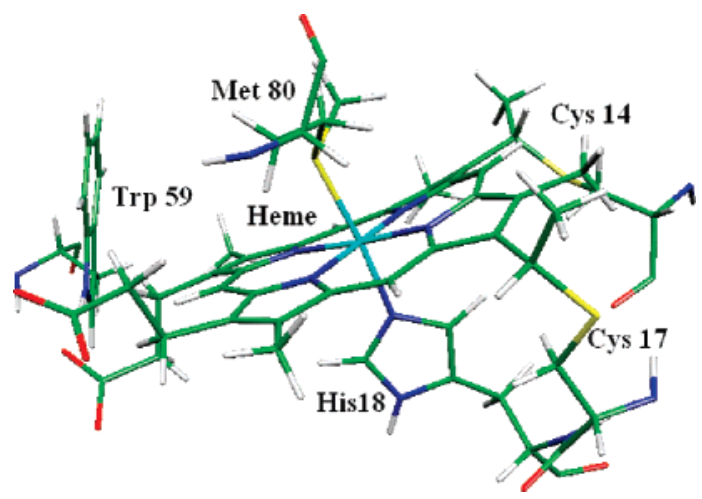

Figure 1. Local structure of the heme group in a native cytochrome c.

\section{Experimental Section}

Cytochrome c (cyt c) from a horse heart in an oxidized form was purchased from the Sigma Chemical Company and used without further purification. Sample solutions containing 21 $\mathrm{mg} / \mathrm{mL}(1.7 \mathrm{mM})$ of cytochrome $\mathrm{c}$, in a buffer solution $(\mathrm{pH} \sim$ 7) of potassium phosphate $(100 \mathrm{mM})$ and $0.1 \mathrm{M} \mathrm{NaCl}$, were prepared with urea in the concentrations of $0,5,6,6.5,7,8$, and $10 \mathrm{M}$ for both SAXS and XAS measurements at $25^{\circ} \mathrm{C}$. For the sake of background subtraction, blank buffer solutions with urea in various concentrations were also prepared.

$\mathrm{X}$-ray absorption spectra for the cytochrome c solutions were measured in a fluorescence mode at the wiggler beamline BL-17C1 of the National Synchrotron Radiation Research Center (NSRRC), Taiwan, with a double crystal monochromator of $\mathrm{Si}(111)$ of an energy resolution, $\Delta E / E$, of $2 \times 10^{-4}$. The energy was scanned from 6.912 to $8.010 \mathrm{KeV}$ using a Lytle detector filled with Ar gas or a 13 element solid-state detector for the near-edge region. A standard Fe foil was used for energy calibration. EXAFS data analysis was completed using the UWXAFS package,${ }^{10}$ with theoretical scattering paths generated by the FEFF7 program ${ }^{11}$ based on model structures. ${ }^{9,15}$

SAXS data were collected using the small-angle X-ray scattering instrument at the beamline 17B3 of NSRRC, with a $0.5 \mathrm{~mm}$ diameter beam of $10 \mathrm{KeV}$. Details of the instrument description were reported previously. ${ }^{12}$ Data measured were then corrected for electronic noise of the area detector, background scattering, incoming flux, sample thickness, and pixel sensitivity of the detector to yield the absolute scattering intensity (scattering cross section per unit sample volume). ${ }^{12}$ Each spectrum of SAXS or XAS presented here was compiled by more than 10 data scans for better statistics. These data scans were quite reproducible, indicating that the radiation damage on the protein was negligible during the XAS or SAXS measurements.

\section{Results and Discussion}

3.1. XAS for Local Structure Changes. Fe K-edge XANES spectra of cytochrome c solutions, both in a native state and in the unfolded state when the $10 \mathrm{M}$ urea is added in the solution, are shown in Figure 2. The spectra are characterized in several regions of $\mathrm{P}(\sim 7113 \mathrm{eV}), \mathrm{A}(\sim 7122 \mathrm{eV}), \mathrm{C}(\sim 7130 \mathrm{eV}), \mathrm{D}$ $(\sim 7140 \mathrm{eV})$, and $\mathrm{C}_{2}(\sim 7153 \mathrm{eV})$, according to a previous XAS study $^{8}$ on a temperature-dependent spin transition of myoglobin. The preedge peak in the $\mathrm{P}$ region is often interpreted as the $1 \mathrm{~s}$ $\rightarrow 3 \mathrm{~d}\left(\mathrm{t}_{2 \mathrm{~g}}, \mathrm{e}_{\mathrm{g}}\right)$ electron quadruple transition, which originates from a distortion of a perfect octahedral symmetry; the distortion increases the $d-p$ mixing of Fe to make the quadruple transition possible. Therefore, the preedge peak is expected to be sensitive to local geometry changes in the coordination of Fe. Regions



Figure 2. XANES spectra of the cytochrome $\mathrm{c}$ both at the native (folded) state (solid line) and in the unfolded state (dashed line) (10 M urea). The inset is the enlarged preedge absorption region.

$A$ and $\mathrm{C}_{2}$ are related to the motion of the iron relative to the porphyrin plane of the heme group, whereas C and D are indicative of the spin state of $\mathrm{Fe}$ as well as the coordination geometry.

As emphasized in the inset of Figure 2, both XANES spectra of the native state and the unfolded state give the same preedge transition in the $\mathrm{P}$ region, indicating that the iron of heme group in this protein maintains the same six-coordinated geometry in both states. Moreover, the significant enhancement in the $\mathrm{C}$ region for the protein in the unfolded state versus the folded state is consistent with a theoretical prediction ${ }^{8}$ based on a spin transition model. In the model, Fe undergoes a spin transition from a low-spin state (LS, $S=1 / 2$ ) to a high-spin state (HS, $S$ $=5 / 2$ ), accompanied by a lengthening of the $\mathrm{Fe}-\mathrm{N} / \mathrm{O}$ bond distance which is responsible for such enhanced absorption in the $\mathrm{C}$ region; by lengthening the $\mathrm{Fe}-\mathrm{N}$ distance, the ligand $2 \mathrm{p}$ orbitals participate less in the hybridization of the $\mathrm{Fe}(4 \mathrm{~s}, 4 \mathrm{p})$ orbitals, leading to the enhancement of the $1 \mathrm{~s} \rightarrow 4 \mathrm{p}$ transition (C region). ${ }^{13}$

Furthermore, the spin transition and the accompanying geometrical change around $\mathrm{Fe}$ can also be interpreted by the coupled changes in the $\mathrm{A}, \mathrm{D}$, and $\mathrm{C}_{2}$ regions shown in Figure 2 , as indicated earlier by Soldatov et al. ${ }^{8}$ and Della Longa et al. ${ }^{7}$ Similar changes in the XANES spectra were also observed for spin-crossover $\mathrm{Fe}(\mathrm{II})$ complexes during spin transition. ${ }^{13}$

To extract more local structure information, we have performed EXAFS data analysis for the XAS spectra of the native and unfolded states (with $10 \mathrm{M}$ urea), as shown in Figure 3a and $\mathrm{b}$ where the weighted EXAFS data $\left(k^{3} \chi\right)$ are illustrated in the insets. The normalized EXAFS spectra are obtained from the absorption data based on the relation $\chi(k)=[\mu(k)-$ $\left.\mu_{0}(k)\right] / \Delta \mu_{0}(0)$, where $\mu(k)$ is the measured absorption coefficient, $\mu_{0}(k)$ the background, and $\Delta \mu_{0}(0)$ the edge jump. The wavenumber $k=\left[2 m\left(E-E_{0}\right) / \hbar\right]^{1 / 2}$ is defined by the photon energy $E$, the threshold energy $E_{0}$, the plank constant $\hbar$, and the mass of the electron $m$. The coordination geometry around Fe obtained from the Fourier transform of $k^{3} \chi$ together with the fitted curve (solid line) for cytochrome $\mathrm{c}$ at the native state are displayed in Figure 3a; details of the fitting procedure used were given in a previous report. ${ }^{14}$ The fitting result includes (1) the first coordination shell of the iron with bond distances, $R$, of 2.00 and $2.10 \AA$, which consists of the four $\mathrm{N}$ (Por) and one $\mathrm{N}$ (His-18) atoms, respectively; (2) the second shell with a characteristic bond distance of $2.41 \AA$ from the $\mathrm{S}$ atom of Met-80; 

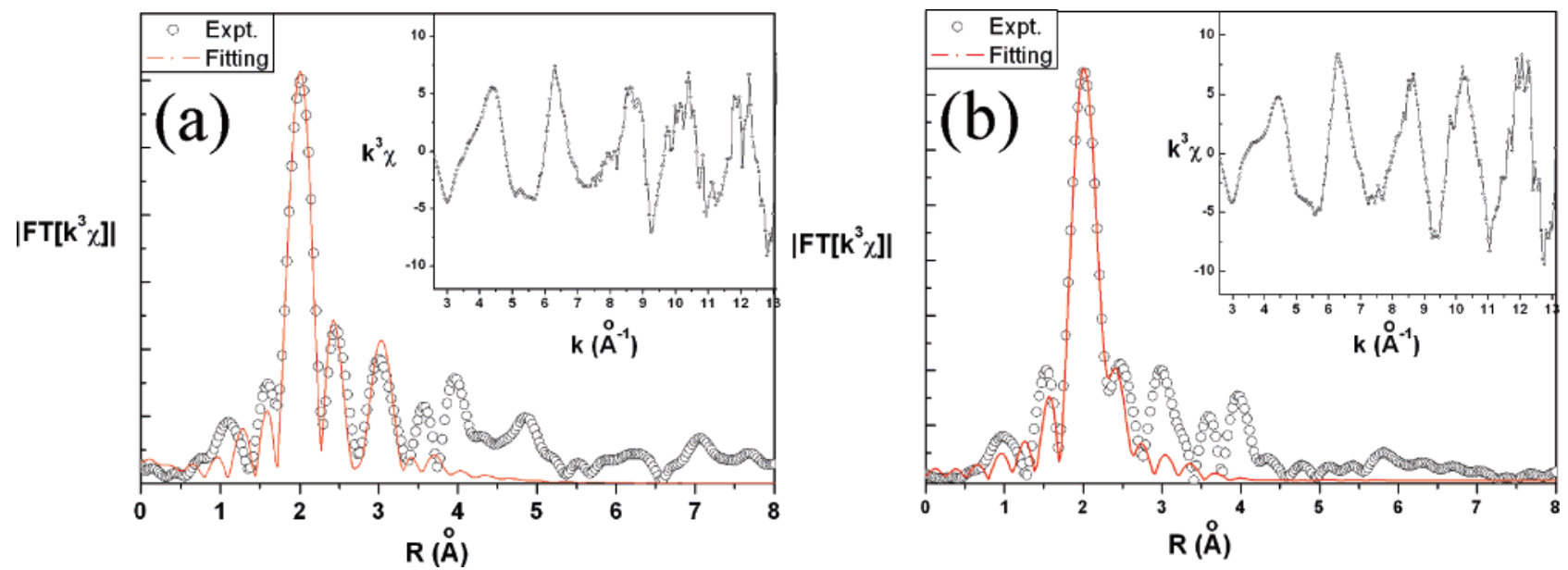

Figure 3. The Fourier transforms (open circle) of the $k^{3} \chi$ data (inset) and the fitted curves (solid line) of (a) the native cytochrome $\mathrm{c}$ and (b) cytochrome $\mathrm{c}$ in $10 \mathrm{M}$ urea.

TABLE 1: EXAFS Fitting Parameters for the Folded and Unfolded States of Cytochrome $\mathrm{c}^{a}$

\begin{tabular}{|c|c|c|c|c|c|}
\hline \multicolumn{3}{|c|}{ folded state } & \multicolumn{3}{|c|}{ unfolded state } \\
\hline bond & $R(\AA)$ & $\sigma^{2}\left(\AA^{2}\right)$ & bond & $R(\AA)$ & $\sigma^{2}\left(\AA^{2}\right)$ \\
\hline $\mathrm{Fe}-\mathrm{N}$ (Por) & $2.00(4)$ & $0.0003(7)$ & $\mathrm{Fe}-\mathrm{N}($ Por $)$ & 2.01(1) & $0.0001(6)$ \\
\hline $\mathrm{Fe}-\mathrm{N}(\mathrm{His})$ & $2.10(4)$ & $0.0003(7)$ & $\mathrm{Fe}-\mathrm{O}$ (water) & $2.25(2)$ & $0.004(3)$ \\
\hline \multirow[t]{2}{*}{$\mathrm{Fe}-\mathrm{S}(\mathrm{Met})$} & $2.41(2)$ & $0.002(2)$ & $\mathrm{Fe}-\mathrm{O}$ (urea) & $2.27(2)$ & $0.004(3)$ \\
\hline & & & $\mathrm{Fe}-\mathrm{C}$ (urea) & $2.60(9)$ & $0.004(3)$ \\
\hline
\end{tabular}

${ }^{a}$ Variable: $R$, interatomic distance; $\sigma^{2}$, mean squared deviation of $R$ obtained from the Debye-Waller factor.

and (3) the third shell with a distance of $3.04 \AA$ from the eight carbon atoms of the porphyrin. The fitted parameters are summarized in Table 1. These bond distances are consistent with those from a previous NMR result ${ }^{9}$ for an average structure of cytochrome $\mathrm{c}$ in the native state, $\mathrm{Fe}-\mathrm{N}($ Por $)=1.980-2.111$ $\AA, \mathrm{Fe}-\mathrm{N}($ His-18) $=1.948 \AA$, and $\mathrm{Fe}-\mathrm{S}($ Met-80) $=2.343 \AA$.

Several possible models were tried for fitting the EXAFS data of the unfolded state; the information of a large global morphology change due to the unfolding of the protein deduced from the SAXS result (see below) does help to eliminate a few models. The final conclusion is a ligand exchange model, with urea and water molecules replacing the two axial ligands of the Met-80 and His-18, respectively. A similar ligand exchange model was also previously proposed by Yeh et al. ${ }^{16}$ in a resonance Raman scattering study on the unfolding of cytochrome $\mathrm{c}$ by a denaturing agent of guanidine chloride. The fitted curve using the ligand exchange model of the unfolding state is shown in Figure 3b. The fitted coordination geometry around $\mathrm{Fe}$ in the unfolding state is with four N(Por) at a bond distance of $2.00 \AA$, which is the same as that in the native state, indicating a relatively stable structure of the porphyrin plane of the heme group after unfolding of the protein. However, the two axial ligands of His-18 and Met- 80 in the folded state are now replaced by the oxygen of water and urea, respectively, with a distance of $2.27 \AA$; an accompanying characteristic bond distance of $R=2.60 \AA$ corresponds to the distance from the $\mathrm{Fe}$ atom to the $\mathrm{C}$ atom of the urea carbonyl group. Of course, the current data cannot completely rule out the possibility of having His-18 remaining in one of the two axial positions in the unfolded state of the protein since there is only one electron difference in $\mathrm{O}$ and $\mathrm{N}$. In this case, only one urea molecule replaces the Met-80 ligand, but with a somewhat lengthened $\mathrm{Fe}-\mathrm{N}$ (His) bond length.

Overall, detailed coordination geometries of Fe in folded and unfolded states obtained from the EXAFS data analysis (Table
1) clearly indicate that during the folding/unfolding process, the local geometry of iron in the heme group changes dramatically along the axial position of the Por plane, namely, the breaking of the Met- 80 ligation, and possibly the His-18, by urea and water molecules, whereas the XANES data reveal a small distortion in the Por plane accompanied by a spin transition of $\mathrm{Fe}(\mathrm{III})$.

3.2 SAXS for Global Structure Changes. Small-angle scattering profiles for colloidal particles of a monodisperse size can be modeled as

$$
I(Q)=I_{0} \tilde{P}(Q) S(Q)
$$

where $\tilde{\mathrm{P}}(Q)$ is the normalized form factor with $\tilde{\mathrm{P}}(0)=1$, and $S(Q)$ is the structure factor with $S(Q) \sim 1$ in a dilute colloidal solution. ${ }^{17}$ The zero angle scattering intensity $I_{0}=C N(b-$ $\left.\rho_{\mathrm{s}} V_{\text {dry }}\right)^{2}$ depends on the concentration $C$, the aggregation number $N$, and the dry volume $V_{\text {dry }}$ for the scattering particles. The scattering length for the particles and scattering length density for the solvent are denoted by $b$ and $\rho_{\mathrm{s}}$, respectively. Without protein aggregation, the expected $I_{0}$ for a cytochrome c solution of $21 \mathrm{mg} / \mathrm{mL}(1.7 \mathrm{mM})$, in the absolute scattering intensity unit, is $0.17 \mathrm{~cm}^{-1}$. With urea added in the aqueous solution, the result is $\rho_{\mathrm{s}}=\left(f_{1} \rho_{1}+f_{2} \rho_{2}\right)$, where $f_{1} \rho_{1}$ and $f_{2} \rho_{2}$ are, respectively, the products of the volume fraction and scattering length density of water $\left(\rho_{1}=9.43 \times 10^{-6} \AA^{-2}\right)$ and urea $\left(\rho_{2}=12.0 \times 10^{-6}\right.$ $\AA^{-2}$ ). An empirical formula by Modig et al. ${ }^{18}$ can be used to calculate the volume fractions of water and urea in the sample solutions prepared. Since the scattering length density of cytochrome c $\left(\rho \sim 12.0 \times 10^{-6} \AA^{-2}\right)$ is very close to that of urea, the increase of urea concentration in a cytochrome $c$ solution reduces the scattering contrast of the protein and, thus, the $I_{0}$ value (or the scattering intensity), as illustrated in the SAXS data shown below.

For homogeneous ellipsoids with semi-major axis $a$ and semiminor axis $b$, the scattering form factor averaged for spatial orientation is given by

$$
\tilde{\mathrm{P}}(Q)=\int_{0}^{1}\left|\frac{3 j_{1}(v)}{v}\right|^{2} \mathrm{~d} \mu
$$

where $v=Q\left[A^{2} \mu^{2}+B^{2}\left(1-\mu^{2}\right)\right]^{1 / 2}$ and $j_{1}$ is the spherical Bessel function of the first order. ${ }^{17}$ For macroion solutions, the structure factor $S(Q)$ depends on the volume fraction $\eta$ and the fractional ionization of the ionic aggregates. ${ }^{17}$ Nevertheless, in buffer solution with salt, the charge interaction between cytochrome 


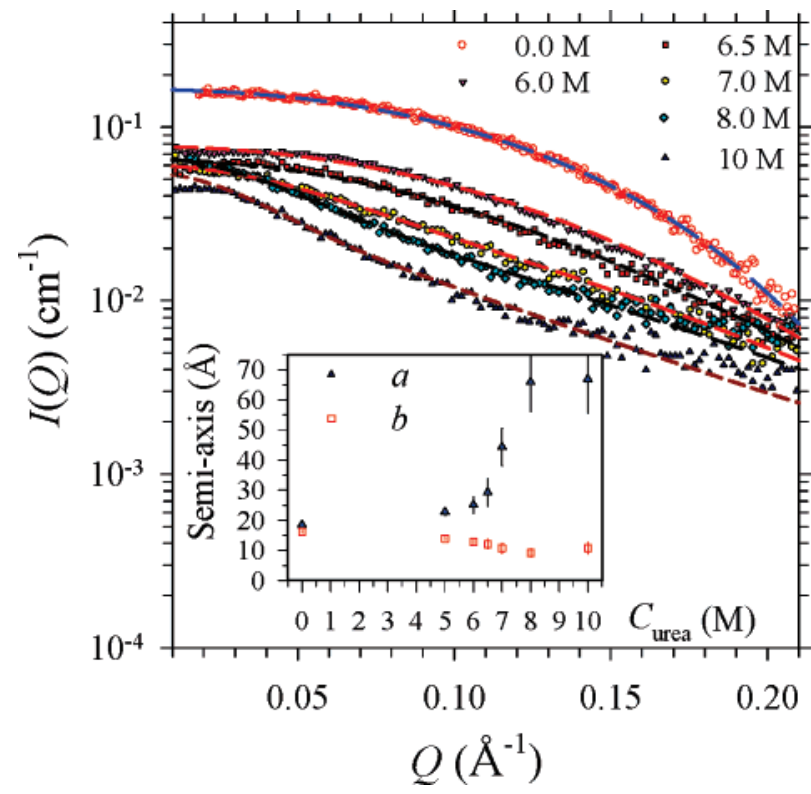

Figure 4. The SAXS data for the cytochrome c solutions, with several urea concentrations from 0 to $10 \mathrm{M}$, are fitted (dashed curves) using the ellipsoid form factor together with the PY structure factor. The inset shows the corresponding values for the semi-axes $a$ and $b$ extracted from the model fitting.

c can be largely screened, and the structure factor $S(Q)$ derived from the Percus-Yevick (PY) model of hard sphere interactions, based on the volume fraction $\eta=C V I N(\sim 1.6 \%$ volume fraction in our case) and an effective sphere diameter $\sigma$ approximated using $V_{\text {dry }}=4 / 3 \pi a b^{2}=4 / 3 \pi(\sigma / 2)^{3}$, may be used to approximate the interactions between cytochrome $\mathrm{c}$ in a buffer solution. ${ }^{19}$ One merit of using the $S(Q)$ from the PY model in a SAXS data fitting process lies in the fact that there is no additional parameter needed in the fitting process, other than that required by the form factor $P(Q)$.

Selectively shown in Figure 4 are the SAXS data measured for the cytochrome $\mathrm{c}$ solutions with $0,6.0,6.5,8.0$, and $10 \mathrm{M}$ urea. All of the data can be fitted systematically at the absolute intensity scale using the ellipsoid model for the form factor $P(Q)$ and the Percus-Yevick model for the structure factor $S(Q)$. The fitted semi-axis lengths $a$ and $b$ shown in the inset indicate that the native cytochrome $\mathrm{c}$, of an aspect ratio (defined by $a / b$ ) of nearly unity, is essentially globular. The globular shape is stable up to a urea concentration of $\sim 5 \mathrm{M}$. Then, the envelope elongates significantly to an ellipsoid-like shape of an aspect ratio $(a / b)$ of $\sim 6$, at $10 \mathrm{M}$ urea. From the fitted semi-axes lengths, we can deduce a radius of gyration $R_{\mathrm{g}}=12.8 \AA$ for the native protein and an $R_{\mathrm{g}}$ value of $29.7 \AA$ for the unfolded protein with $10 \mathrm{M}$ urea. ${ }^{17}$

\section{Discussion}

On the basis of the XAS and SAXS results, it is clear that cytochrome $\mathrm{c}$ undergoes a significant local and global structural transition during the unfolding process introduced by urea. Articulating the local and global structural changes observed, a possible unfolding route for the protein in urea solution is proposed: the approach of the urea molecule toward the heme group may induce a spin transition of the iron in the heme group from a LS to a HS state, thus weakening and breaking the bonds of $\mathrm{Fe}-\mathrm{S}(\mathrm{Met}-80)$ and/or $\mathrm{Fe}-\mathrm{N}(\mathrm{His}-18)$, leading to a ligand exchange of urea and/or $\mathrm{H}_{2} \mathrm{O}$. Although we cannot completely rule out the possibility with water binding due to the weak effect of the second shell, the EXAFS fitting with urea, including the
$\mathrm{C}$ atom of urea to distinguish from water binding at the axial position, is better. A similar solvent effect on a spin transition of $\mathrm{Fe}$ (II) complexes was also observed in a previous report. ${ }^{20}$ In view of the molecular structure of cytochrome $\mathrm{c}$, the heme can be a node for holding the globular structure of the protein, and the breaking of the axial ligands (observed by XAS) may serve as a key factor for unfolding the protein from a globular envelope to an elongated ellipsoid-like morphology (observed by SAXS). A systematic study, with combined XAS and SAXS, on the cytochrome $c$ in various concentrations of urea is definitely useful for understanding the details of the unfolding process of the protein, for instance, the sequential unfolding process proposed recently by Maity et al. ${ }^{21}$

Furthermore, since the spin transition of iron from the LS to HS state could lead to a deformation of the porphyrin ring, it is possible to examine the spin transition due to the unfolding of cytochrome $\mathrm{c}$ by the changes of resonance Raman spectra in the region of $\sim 1000-1700 \mathrm{~cm}^{-1}$, a region that carries the characteristic $\mathrm{C}-\mathrm{N} / \mathrm{C}-\mathrm{C}$ vibrations of the porphyrin ring. The two vibration bands at 1584 and $1566 \mathrm{~cm}^{-1}$ are considered to be especially sensitive to the spin transition of Fe during the unfolding process of protein. ${ }^{22-23}$

\section{Conclusions}

The systematic study, based on combined XAS and SAXS, on the folding/unfolding of cytochrome $\mathrm{c}$ in the presence of urea leads to a ligand exchange model, with urea and/or water to replace the Met- 80 and/or His-18 ligands of iron in the heme group. The XANES and EXAFS of native and unfolded cytochrome $\mathrm{c}$ measured at the Fe K-edge give direct evidence of spin transition in the $\mathrm{Fe}$ of the heme group together with significant changes in the ligand geometry, including the replacement of the Met-80 and/or His-18 ligand, for the unfolding process. The SAXS result reveals a drastic change in the protein morphology from a globular to an ellipsoidal shape during the unfolding process. A plausible mechanism for the unfolding of cytochrome $\mathrm{c}$ is that the spin transition of Fe takes place first in the presence of urea, followed by a ligand exchange from Met- 80 and/or His-18 to urea and/or water molecules. Of course, the accompanied morphology change occurs when the Met-80 leaves the coordination of Fe. It is proven that an integrated study of XAS and SAXS can provide the necessary information of the unfolding process of a protein.

Acknowledgment. We thank Drs. H. S. Sheu and J. J. Lee for the discussion, Mr. C.-C. Tseng at the NSRRC for preparing part of the sample solutions, and Mr. C.-W. Pao for assisting with the XAS measurement. The work was partially supported by the NSC under Grant No. NSC-95-2112-M-213-002.

\section{References and Notes}

(1) Wu, H. Chin. J. Physiol. 1991, 5, 321-327.

(2) Ibarra-Molero, B.; Sanchez-Ruiz, J. M. Biochemistry 1997, 36, 9616-9624.

(3) (a) Doniach, S. Chem. Rev. 2001, 101, 1763-1778. (b) Cinelli, S.; Spinozzi, F.; Itri, R.; Finet, S.; Garsughi, F.; Onori, G.; Mariani, P. Biophys. J. 2001, 81, 3522-3533.

(4) Koch, M. H. J.; Vachette, P.; Svergun, D. I. Q. Rev. Biophys. 2003 , $36,147-227$

(5) Uzawa, T.; Akiyama, S.; Kimura, T.; Takahashi, S.; Ishimori, K.; Morishima, I.; Fujisawa, T. Proc. Natl. Acad. Sci. U.S.A. 2004, 101, $1171-$ 1176.

(6) Lipfert, J.; Doniach, S. Annu. Rev. Biophys. Biomol. Struct. 2007, $36,307-327$

(7) Boffi, F.; Bonincontro, A.; Cinelli, S.; Castellano, A. C.; De Francesco, A.; Della Longa, S.; Girasole, M.; Onori, G. Biophys. J. 2001, $80,1473-1479$. 
(8) (a) Soldatov, A. V.; Yalovega, G. E.; Smolentsev, G. Yu.; Kovtun, A. P.; Della Longa, S. Biophysics 2001, 46, 564-568. (b) Della Longa, S.; Pin, S.; Cortès, R.; Soldatov, A. V.; Alpert, B. Biophys. J. 1998, 75, 31543162 .

(9) Banci, L.; Bertini, I.; Gray, H. B.; Luchinat, C.; Reddig, T.; Rosato, A.; Turano, P. Biochemistry 1997, 36, 9867-9871.

(10) (a) Frenkle, A. I.; Stern, E. A.; Voronel, A.; Qian, M.; Newville, M. Phys. Rev. B 1994, 49, 11662-11665. (b) Newville, M.; Ravel, B.; Haskel, D.; Rehr, J. J.; Stern, E. A. Physica B 1995, 208, 154-159.

(11) Zabinsky, S. I.; Rehr, J. J.; Ankudinov, A.; Albers, R. C.; Eller, M. J. Phys. Rev. B 1995, 52, 2995-2999.

(12) Lai, Y. H.; Sun, Y. S.; Jeng, U.; Lin, J. M.; Lin, T.-L.; Sheu, H.S.; Chuang, W.-T.; Huang, Y.-S.; Hsu, C.-H.; Lee, M.-T.; Lee, H.-Y.; Liang, K. S.; Gabriel, A.; Koch, M. H. J. J. Appl. Crystallogr. 2006, 39, 871-877. (13) (a) Briois, V.; dit Moulin, Ch. C.; Sainctavit, Ph.; Brouder, Ch.; Flank, A.-M. J. Am. Chem. Soc. 1995, 117, 1019-1026. (b) Real, J. A.; Castro, I.; Bousseksou, A.; Verdaguer, M.; Burriel, R.; Miguel Castro, M.; Linares, J.; Varret, F. Inorg. Chem. 1997, 36, 455-464. (c) Lee, J. J.; Sheu, H. S.; Lee, C. R.; Chen, J. M.; Lee, J. F.; Wang, C. C.; Huang, C. H.; Wang, Y. J. Am. Chem. Soc. 2000, 122, 5742-5747. (d) Okamoto, K.; Nagai, K.; Miyawaki, J.; Kondoh, H.; Ohta, T. Chem. Phys. Lett. 2003 371, 707-712. (e) Oyanagia, H.; Tayagakib, T.; Tanaka, K. J. Phys. Chem Solids 2004, 65, 1485-1489. (f) Khalil, M.; Marcus, M. A.; Smeigh, A. L.; McCusker, J. K.; Chong, H. H. W.; Schoenlein, R. W. J. Phys. Chem. A 2006, 110, 38-44.

(14) Hsu, I. J.; Liu, R. S.; Chen, J. M.; Liu, R. G.; Jang, L. Y.; Lee, J. F.; Harris, K. D. M. Chem. Mater. 2000, 12, 1115-1121.
(15) The codes JAJJOU, MANJIU, and MITQEV in the Cambridge database are used to assist the building of the urea replacement model.

(16) (a) Yeh, S. R.; Takahashi, S.; Fan, B.; Rousseau, D. L. Nat. Struct Biol. 1997, 4, 51-56. (b) Takahashi, S.; Yeh, S. R.; Das, T. K.; Chan, C. K.; Gottfried, D. S.; Rousseau, D. L. Nat. Struct. Biol. 1997, 4, 44-50. (c) Yeh, S. R.; Rousseau, D. L. J. Biol. Chem. 1999, 274, 17853-17859. (d) Yeh, S. R.; Han, S.; Rousseau, D. L. Acc. Chem. Res. 1998, 31, 727736.

(17) Chen, S. H.; Lin, T. L. Methods of Experimental Physics. Neutron Scattering in Condensed Matter Research; Sköd, K., Price, D. L., Eds.; Academic Press: New York, 1987; Vol. 23B, Chapter 16.

(18) Modig, K.; Kurian, E.; Prendergast, F. G.; Halle, B. Protein Sci. 2003, 12, 2768-2771.

(19) Hayter, J.; Penfold, J. Mol. Phys. 1981, 42, 109-114.

(20) (a) Halder, G. J.; Kepert, C. J.; Moubaraki, B.; Murray, K. S.; Cashion, J. D. Science 2002, 298, 1762-1765. (b) Niel, V.; Thompson, A. L.; Carmen Muñoz, M.; Galet, A.; Goeta, A. E.; Real, J. A. Angew. Chem., Int. Ed. 2003, 42, 3763-3766. (c) Glaser, T. Angew. Chem., Int. Ed. 2003, 42, 5668-5670.

(21) Maity, H.; Maity, M.; Englander, S. W. J. Mol. Biol. 2004, 343, 223-233.

(22) (a) Spiro, T. G.; Strekas, T. C. J. Am. Chem. Soc. 1974, 96, 338345. (b) Spiro, T. G.; Strekas, T. C. Proc. Natl. Acad. Sci. U.S.A. 1972, 69, 2622-2626. (c) Jordan, T.; Eads, K. C.; Spiro, T. G. Protein Sci. 1995, 4 $716-728$.

(23) Yamamoto, T.; Palmer, G.; Gill, D.; Salmeen, T.; Rimai, L. J. Biol. Chem. 1973, 248, 5211-5213. 